\title{
Commentary on the Special Issue
}

\author{
Gary D. Phye
}

Published online: 16 August 2012

(C) Springer Science+Business Media, LLC 2012

At the outset, I must confess to being very enthusiastic about the line of research being presented. On a larger scale, I am very pleased to see experimental psychology as conducted by applied cognitive scientists focusing on principles of learning, memory, and cognition that have implications for classroom practice. At the same time, these principles, generally speaking, have been available to classroom teachers in one form or another for some time. The question that I keep asking myself is "what didn't work before, what went wrong?" Why has there been no wide spread adoption of these learning principles by educational professionals?

Prior to specific comments about individual contributions, I am going to take the liberty of engaging in some philosophical thoughts. First, I will comment on a couple of metaphors frequently employed by educators when making meaning about educational progress and reform. Second, I will recommend the consideration of "the correspondence principle" as described by Gibson (1994) as a means of facilitating communication among researchers and practitioners. Following comments on individual articles, I suggest the development of a model-based reasoning approach (Mislevy 2009), that should not only enhance the construct validity of the arguments presented, but enhance communication between basic researchers and educational professionals. Models, like metaphors, provide a common referent for communication and are a critical component in the conventionalization process.

\section{Metaphors and Models}

When educational psychologists discuss the history of implementing educational practices (innovations?) in the classroom, reference is frequently made to "pendulum swings". This metaphor provides discussants with a visual image meant to portray the changes from one theoretical extreme to another that occur in many curriculum content areas. Another way in which educational psychology has characterized change in educational settings is the use of the biological principle of epigenesis. This principle provides the discussants with an image

G. D. Phye $(\bowtie)$

N162B Lagomarcino, Iowa State University, Ames, IA 50011-3196, USA

e-mail: gdphye@iastate.edu 
of recursive growth. This image serves as the basis for the concept of "spiral curriculum" as introduced by Bruner (1960) in his effort to make meaning of how instruction and the curriculum provide guidance in the development of personal academic knowledge of students. In both cases, communication among educational researchers and scholars has been shaped by the use of these metaphors. Examples coming quickly to mind are mathematics and reading. Interestingly, these two content areas are required by the federal government for accountability purposes to which schools in the USA are held.

In some respects, it could be argued that the images of pendulum swings and a spiral curriculum characterize many educational researchers' view of what Kuhn (1962) identified as elements of a "paradigm shift". While Kuhn was speaking to all scientists and science in general, the essence of Kuhn's work, as I read it, was to characterize the way in which a community of scientists communicate, engage in theoretical dialog, etc. and how the discipline moves forward in an effort to better understand the discipline in terms of precision and explanation. There are several elements involving a paradigm shift that are unique to a discipline. These elements may facilitate or impede progress.

\section{Level of Analysis and the Correspondence Principle}

Gibson (1994), whose audience was psychologists, addressed the lack of communication among basic and applied psychologists. Her point was that different specialties in psychology address the same human behavior from quite different levels of observation and analysis but that there should be a correspondence among levels in terms of research findings. This is not a reductionist view proposing a cause-effect relationship from brain cell to classroom performance (grade card), that is prescriptive in nature. Rather, the issue is simply a correspondence between and among levels of analysis. For example, educational researchers studying classroom learning might take a constructivist perspective in their study of academic learning. This perspective might be quite global with a function of studying student academic growth (learning) during the fourth grade. Little effort may be made to analyze with precision, elements contributing to the academic growth (instructional situations, types of assessments, individual differences, etc.). Consequently, one would characterize the level of analysis as global. Change that is a critical element in any definition of classroom learning would be observed, but if the data are too global, it is difficult to develop effective educational innovations or practice. Much of this research is conceptualized as moving theory to practice. Current emphasis on educational reform speaks to the limited success of past efforts.

At a more precise level of analysis and explanation, one can locate the applied cognitive psychologist (current contributors) providing research evidence for the development of "principles of learning, memory and cognition" that have classroom application. Theoretically, it is difficult to ignore the suggestive nature of the data presented in the current articles. This line of research has been identified as the functional study of memory and cognition and can be viewed as a moderate level of precision and analysis in the study of learning memory and cognition. Unfortunately, there is a scarcity of testable models that would serve as the basis for model-based reasoning, which is critical for communication across disciplines. If there is little ability to communicate, there is little likelihood of successful implementation in the classroom. This communication problem is recognized by Karpicke and Grimaldi (this issue) with reference to Pasture's Quadrant.

I am suggesting that a three-tier level of analysis implicitly exists in the study of learning, memory, and cognition (educational research, educational psychology, applied cognition). I am 
sure that someone with a better understanding of research in these areas could propose a four- or five-tier model of analysis. I view the current efforts of Educational Psychology Review's editorship team to be a step in the direction of enhancing the communication among the aforementioned research communities. The authors' of the articles in this special issue are to be commended for their willingness and efforts to partake in this endeavor to enhance the communication process among researchers with a common interest in classroom learning.

\section{Commentary}

\section{Son and Simon}

Son and Simon provide a nice review of both the current status of distributed learning as well as highlights of previous research in verbal and motor learning. The implications of spacing and potential contributions to the study of metacognition are less convincing. This may be due in part to the greater differences in the operational definition of what constitutes valid measures of metacognitive functions in education and psychology. The reminder of the impact of distributed learning involving motor learning is a major contribution and a reminder to educators that many classroom experiences involve motor learning. Not all of our public school students are college bound and there are many K-12 educational experiences that involve learning technical skills with motor learning components.

I see the educational experiences of cognitively challenged students benefitting from distributed learning experiences that are incorporated into the development of individual education programs. Also, remedial programs where attention is being focused on moving nonproficient students in math and reading to a higher skill level of proficiency would also benefit. Any study skills program where time management is taught as part of the selfregulation would also appear to be a target for research efforts combining spacing and metacognitive monitoring principles.

Marsch, Butler, and Umanath

The Marsch, Butler, and Umanath article is a research area of which I was unaware. I was extremely excited about the potential of this line of research impacting the reading research efforts in education and educational psychology. The emphasis on prior knowledge and source memory is a very creative approach to the question of using fiction to teach reading at the elementary and middle school levels. The fact that there are inaccuracies in fiction, as well as lesser cognitive processing depth when engaging in reading for pleasure, has curriculum implications. The trade-off of motivation and interest for lesser cognitive effort (when reading fiction) may well be contributing to the large numbers of college students we are currently seeing in the classroom who are reading at the ninth- or tenth-grade levels. I also see a great interdisciplinary opportunity for collaborative research efforts in science education where misconception research has a high priority. In many respects, this line of research appears to me to be as transportable at the present time as any of the research addressed in this special issue.

Carpenter, Cepeda, Rohrer, Kang, and Pashler

The Carpenter et al. article provides the reader with the next logical step in progressively expanding data collection efforts to include diverse forms of learning more characteristic of 
classroom conditions. Also, as teachers, we all deal with teaching episodes that extend across a semester or an academic year. Consequently, we are all concerned with the long-term retention of content we teach. This applies not only with concern about what is remembered during and at the end of the courses we teach, but the question of what is remembered (reconstructed) and used in following courses to accommodate new learning efforts (positive transfer?). As pointed out in this article, a comprehensive final exam (of some form) is required to promote long-term retention during classroom learning. This assessment practice coupled with the "method of savings" logic provides an explanation for the positive impact on relearning of concepts and facts that are beyond recall as the semester progresses and new material is encountered. To this educational psychologist, the extension of learning materials to classroom mathematics and the findings obtained, have serious implications for further classroom research on the spacing of teaching activities during the academic year.

\section{Rohrer}

The Rohrer article is very well written. It clarifies and extends an extremely important issue involving the spacing effects for promoting long-term retention that were discussed in the Carpenter et al. article. This issue is one of how to re-expose students to prior concepts and cognitive procedures taught earlier in the course. In other words, are there different ways to structure practice (relearning) such that higher order thinking skills (procedural knowledge) are being promoted as well as basic relearning of concepts and facts (declarative knowledge).

The instructional goal of most teachers today is to promote both a factual and conceptual foundation for the development of declarative knowledge and the ability to use higher order cognitive operations (procedural knowledge) to think critically and solve problems. There are several ideas provided by Roher that will facilitate the achievement of this goal. The discussion of discrimination learning is right on target but only half of the picture when promoting the higher order thinking skills of analysis, synthesis, and evaluation required for problem solving. The rest of the story is generalization learning.

Research involving cognitive training has made a strong case (Klauer and Phye 1994, 2008; Phye and Johnson 2009), for teaching both discrimination and generalization as cognitive processing skills critical to the development of inductive reasoning ability that promotes problem solving transfer. The facilitative effect of cognitive training appears to impact the first two phases (problem identification and problem representation) of the problem solving process. A review of approximately 80 research studies (Klauer and Phye 2008) has demonstrated the effectiveness of teaching both discrimination and generalization processing when working with a large cross-section of students in varied settings.

In the studies reviewed by Klauer and Phye (2008), all followed a program format consisting of 12 training sessions over a 3- to 4-week period with a total of 120 problem solving episodes. Each training session consisted of 10 problems. Problem solutions across the 12 training sessions required generalization learning, discrimination learning, and a combination of generalization and discrimination. These cognitive skills were employed in problem solutions involving attributes of the stimulus materials or problem solutions involving relationships of the stimulus materials. The 12 training sessions, each with its 10 problems, follows a hybrid interleaving format ordered in difficulty of cognitive processing (generalization then discrimination, then the combination) as well as the nature of the material (attributes then relations). The hybrid interleaving format in the cognitive training program promotes the durability of problem solving and is commonly maintained over a 6-month interval. 
There are a number of different cognitive training programs that have been employed in educational settings across Western and Eastern Europe. These programs are slowly gaining visibility in the USA. This would appear to be a natural fit for collaborative efforts involving the interleaving effects of practice. I would also suggest that interleaving effects of classroom formative assessments across the academic year will be greatly impacted by the effective implementation of interleaving principles in the development of formative assessment schedules.

\section{Rawson and Dunlosky}

The Rawson and Dunlosky article is very well written and presents a nicely integrated perspective on testing effects. This article serves as an integration of many of the basic principles of memory retrieval in a manner that will resonate with educational researchers. The emphasis on effectiveness/efficiency and durability of test-enhanced learning fits well with the work of educational psychologists focusing on efficiency in learning and the management of cognitive load during training (Clark et al. 2006). This article will be very attractive to the educational researchers focusing on instructional models for the classroom.

The relearning model has many positive attributes which make it attractive to classroom teachers. However, as pointed out by the authors, the enigma facing researchers are the scaling issues involved with measurement. The quandary involves the fact that as learning episodes vary in size and scope, proportions of the same value means quite different things. This is a characteristic of any ordinal measurement scale and makes generalizability to the level of principles quite difficult. A statistical transformation of the data to the level of an interval scale would improve the generalizability of results. Given the psychometric principle of individual differences (both inter-individual and intra-individual), the logic of a ratio scale for describing optimal relearning intervals in terms of proportions is simply not available from a measurement perspective. Thus, mapping an optimal relearning schedule would be a daunting task. However, given the track record of the authors, I have confidence that they will make considerable inroads into the solution of this problem.

\section{Karpicke and Grimaldi}

I view the Karpicke and Grimaldi perspective as different from the other articles in two ways. The scope of the proposed model involves meaningful learning and the emphasis on reconstruction as a critical feature in retrieval based learning. This approach to the testing effect will resonate positively in educational circles where formative assessment is viewed as an integral part of the classroom learning process. Secondly, I more clearly see these data as part of an assessment model rather than an instructional model.

I base these assertions on the author's definition of meaningful learning. Meaningful learning is thought to produce organized, coherent, and integrated mental models that allow people to make inferences and apply knowledge. As the current authors state, "it is important to remember that in all circumstances people transfer past experiences to meet the demands of the present, so all circumstances involve reconstructing knowledge based on cues available in a particular retrieval context". "Regardless of whether the goal of retrieval is to recall a fact, make an inference, or solve a new problem, the ability to accomplish the task depends on using retrieval cues to reconstruct knowledge". Thus the act of retrieval itself is central to the learning process and the development of a personal knowledge base that promotes meaningful learning. 
This line of research will benefit greatly from the widespread adoption of the core curriculum by the majority of states in the USA. The core curriculum provides meaningful student learning outcomes for each grade level. These meaningful learning outcomes are operationally defined in terms of both major concepts and cognitive processing skills. Consequently, basic researchers seeking to impact classroom learning practices can more easily make the case for the construct validity of the models being developed.

Agarwal, Bain, and Chamberlain

I saved my comments for the Agarwal, Bain, and Chamberlain article for last because of the hope it inspired in me that this time we may get it right in term of the implementation of basic learning principles. The involvement of both teachers and building administration is a critical factor in the successful implementation of the principles discussed herein. When there is a collaborative partnership between researchers and educational personnel, a mutual appreciation develops that not only promotes successful implementation, but also provides researchers with insight into the complexities of classroom environments.

My only concern is the small number of students impacted over a 5-year period. This is not a negative comment. Rather, it is a concern for how these very positive efforts can be disseminated on a broad scale. The Agarwal et al. article is a positive story about facilitating educational reform. In this respect, the following comments based on Mislevy's recent work are offered in the spirit of enhancing the development of a research culture that may contribute to our "scaling up" of such efforts.

With no comprehensive answer to the scaling up problem, I suggest we start with the communication process. Too many educators and educational psychologists envision a twostep process from theory to practice. Most applied cognitive psychologists either implicitly or explicitly proceed on the basis of a three-step process; (1) theory, (2) model, and (3) practice. My experience has been that when experimental psychologists and educational psychologists communicate through the journals (Pasteur's Quadrant?), this discrepancy in the use of models impedes successful communication because discussants are talking about the same phenomenon at different levels of analysis. Consequently, our effort to move theoretical principles directly to practice with fidelity of implementation is extremely rare.

\section{Model-Based Reasoning in Science}

At the beginning of my commentary, I made reference to renewed interest in model-based reasoning by educational psychologists studying measurement issues. My earlier mention of communication within Pasteur's Quadrant is the context for the following comments based on the description of Mislevy (2009) of aspects of model-based reasoning.

- While there is an applied cognitive model being implicitly articulated by the contributing authors that characterizes model formation, an explicit articulated model elaborating the correspondences across overlapping models would greatly benefit educational researchers (horizontal correspondence).

- An explicitly articulated model would enhance communication and collaborative research efforts among educational psychologists and applied cognitive experimental psychologists (vertical correspondence). Consequently, this should enhance the validity of implementation efforts by educational psychologists who are involved with: (1) model evaluation (assessing the correspondence between model components and their real 
world counterparts); (2) model revision (modifying or elaborating a model to better establish a correspondence between levels of analysis).

- These efforts to enhance the communication process could produce a shared research culture identified by Mislevy as model-based inquiry with researchers and educational professionals working interactively between phenomena and models with an emphasis on monitoring and using real world feedback in the development of educational policy and practice in the classroom.

As mentioned at the outset, I believe that the current contributions focusing on the application of cognitive principles in the classroom have great potential for the development of both classroom instructional models and classroom formative assessment models. Critical for both collaborative research efforts and the fidelity of classroom implementation by educational psychology and educational professionals is the employment of model-based reasoning.

\section{References}

Bruner, J. (1960). The process of education. Cambridge: Harvard University Press.

Clark, R., Nguyen, F., \& Sweller, J. (2006). Efficiency in learning: Evidence-based guidelines to manage cognitive load. San Francisco: Pfeiffer.

Gibson, E. J. (1994). Has psychology a future? Psychological Science, 5, 69-76.

Klauer, K. J., \& Phye, G. D. (1994). Cognitive training for children: A developmental program of inductive reasoning and problem solving. Seattle: Hogrefe \& Hubner.

Klauer, K. J., \& Phye, G. D. (2008). Inductive reasoning: A training approach. Review of Educational Research, 78(1), 85-123.

Kuhn, T. S. (1962). The structure of scientific revolutions. Chicago: University of Chicago Press.

Mislevy, R. J. (2009). Validity from the perspective of model-based reasoning. In R. L. Lissitz (Ed.), The concept of validity: Revisions, new directions and applications. Charlotte: Information Age.

Phye, G. D., \& Johnson, S. A. (2009). Cognitive training: Improving problem-solving transfer skills of learning students. Educational \& Child Psychology, 26(3), 59-70. 